\title{
Acquisition of the lac operon by Salmonella enterica
}

\author{
Susan R. Leonard, David W. Lacher and Keith A. Lampel
}

\begin{abstract}
Background: Classical bacteriological characteristics of Salmonella enterica indicate that the members of this species are unable to utilize lactose as a carbon source. However, lactose-fermenting (Lac+) strains of several Salmonella serovars have been isolated from different foodborne outbreaks as well as different geographical regions worldwide. In the present study, we sequenced the genomes of $13 \mathrm{Lac}+$ S. enterica isolates and characterized the lac region, comparing it to the lac region in other enteric bacterial species.

Results: Genetic analysis of the lac operons in the S. enterica genomes revealed that they all contain intact lacl, lacZ, and lacY genes. However, lacA was truncated in all of the S. enterica subsp. enterica isolates, encoding a 56 amino acid peptide rather than the full length 220 amino acid LacA protein. Molecular analyses of the 13 isolates revealed that the lac operon resided on a plasmid in some strains and in others was integrated into the bacterial chromosome. In most cases, an insertion sequence flanked at least one end of the operon. Interestingly, the S. enterica Montevideo and S. enterica Senftenberg isolates were found to harbor a plasmid with a high degree of sequence similarity to a plasmid from Klebsiella pneumoniae strain NK29 that also harbors the lac operon. In addition, two S. enterica Tennessee isolates carried two copies of the lac operon. Phylogenetic analysis based on laclZY gene sequences determines distinct clusters, and reveals a greater correlation between laclZY sequence and flanking organization than with either bacterial species or genomic location.

Conclusions: Our results indicate that the lac region is highly mobile among Enterobacteriaceae and demonstrate that the Lac $+S$. enterica subsp. enterica serovars acquired the lac region through parallel events. The acquisition of the lac operon by several $S$. enterica serovars may be indicative of environmental adaptation by these bacteria.
\end{abstract}

\section{Background}

Salmonella enterica consists of over 2500 serovars, most of which are non-typhoidal and invade the host intestinal epithelium resulting in enterocolitis/diarrhea $[1,2]$. Transmission of non-typhoidal S. enterica occurs predominately through contaminated food or water, but also spreads by person to person contact, or contact with infected animals $[1,2]$. Lactose-fermenting ability is a biochemical test often used diagnostically to differentiate $S$. enterica from other Enterobacteriaceae, particularly Escherichia coli, as the genes responsible for conferring the lactose-fermenting phenotype are not harbored by most S. enterica strains [3, 4]. However, although infrequent, outbreaks caused by lactose-fermenting (Lac+) $S$. enterica have been reported [5-7], including a recent

\footnotetext{
* Correspondence: Keith.Lampel@fda.hhs.gov

Division of Molecular Biology, Center for Food Safety and Applied Nutrition, U.S. Food and Drug Administration, Laurel, MD, USA
}

outbreak due to contamination of peanut butter with $S$. enterica Tennessee that sickened 715 individuals [8]. Occasional incidences of Lac $+S$. enterica have been reported for a variety of serovars [9-16].

The lac operon, comprised of the genes lacZ, lacY, and $l a c A$, encodes the proteins responsible for lactose utilization. LacY, lactose permease, transports lactose into the cytoplasm where LacZ, $\beta$-galactosidase, cleaves lactose into glucose and galactose. The lacA gene encodes a transacetylase that is not an essential requirement for lactose catabolism. The lac region also includes the lacI gene, located just upstream of lacZ, that encodes a repressor that regulates transcription of the lac operon. While the lac region is located in the E. coli chromosome, it has been reported to be carried on a plasmid in several Enterobacteriaceae including two serovars of S. enterica [12]. Cornelis et al. determined that the lacI, lacZ, and lacY genes, along with flanking 
sequence, are found on a plasmid-encoded transposon, Tn951, in a Lac + strain of Yersinia enterocolitica. [17]. Isolation of a Lac + plasmid from S. enterica Typhi has been reported and an insertion sequence (IS) element, IS1, was revealed to be in the vicinity of the lac region on the plasmid [14]. However, in general, only phenotypes have been reported for Lac $+S$. enterica with no accompanying sequence or genetic analysis.

Horizontal transfer of transmissible elements has played a major role in the acquisition of new genetic information by bacterial species. The underlying physiological explanations for the acquisition or loss of genetic information, such as the lac operon, are not always apparent. Loss of genetic information may enhance virulence, and in other situations, would improve the bacterium's ability to adapt to its environment (host). Characterization of the lac region could aid in understanding how the lac operon is transferred between enteric bacteria, including $\mathrm{Lac}+S$. enterica isolates. In the current study, we compare the lac region and flanking sequence of the genome in 13 lactose-fermenting $S$. enterica isolates belonging to five different serovars along with other enteric bacterial species. Although a number of Lac + Salmonella strains have been identified since the turn of the $20^{\text {th }}$ century [11], a detailed comparison of this region in representatives in this genus has not been reported. Several of the isolates chosen in this study either originated in regions where $\mathrm{Lac}+$ Salmonella are more common (S. enterica Montevideo) or isolated from a food-related outbreak (S. enterica Tennessee).

To elucidate a potential transfer mechanism for the lac genes, the regions that surround the lac operon were sequenced to identify any genetic elements that may be involved in the mobilization of these genes. We demonstrated that with the exception of the S. enterica subsp. diarizonae isolates, the lac region is flanked by insertion sequences and that this entire genetic region is itself carried within a mobile genetic element. Whole genome sequence analyses in this study provide a putative mechanism as to the gain of the lac operon via transposable elements. Acquisition of these additional genes may provide the host bacterium, i.e., Lac + Salmonella, advantages to environmental adaptation.

\section{Results}

\section{Molecular serotyping}

The serotypes of the S. enterica isolates were confirmed by molecular means and are listed in Table 1 . Two of the isolates were identified as $S$. enterica subsp. diarizonae. The lactose-fermenting ability exhibited by these isolates is not surprising since $85 \%$ of $S$. enterica subsp. diarizonae are known to be Lac + [18], a much higher percentage than the $0.8 \%$ reported for S. enterica subsp. enterica [4]. The remaining 11 isolates are serovars of S. enterica subsp. enterica, including five Senftenberg, three Tennessee, two Montevideo, and one Indiana. While a Lac + phenotype has been reported occasionally for a variety of S. enterica subsp. enterica serovars [5-9, 12-16], to our knowledge, lactose-fermenting Montevideo strains have not been reported previously.

\section{Lac phenotype}

MacConkey agar plates were examined for red colonies indicating lactose fermentation. All of the S. enterica isolates included in this study displayed a Lac + phenotype, demonstrating that the isolates lacking $l a c A$ retain functional lac operons.

\section{Characterization of the lac operon and flanking regions}

The lac operon regions were analyzed for the presence of the four lac genes as well as the genetic elements that flank the lac region. The results of this analysis are shown in Table 1. Interestingly, it was discovered that the genomes of two of the Tennessee isolates, 50260 and 50261, harbor two lac regions. To distinguish the two lac operons for these isolates, they will be referred to as lac1 and lac2. All 13 S. enterica genomes were found to contain intact lacI, lacZ, and lacY genes. The S. enterica subsp. diarizonae lac regions also contained intact copies of the lacA gene. However, lacA was truncated in all of the $S$. enterica subsp. enterica isolates, encoding a 56 amino acid product rather than the full length 220 amino acid LacA found in E. coli K-12 MG1655. With the exception of the E. coli isolates, this discovery held for several other enteric bacteria examined in this study where lacA was either truncated or completely missing.

Extending the genetic analysis of the S. enterica subsp. enterica isolates to regions adjacent to the lac region revealed the presence of IS elements both upstream of lacI and downstream of the lacA remnant (Table 1). Characterization of the lac operon and flanking regions of other enteric bacterial species revealed that although in some cases the regions include different IS elements, lac operon regions are in almost all cases flanked by IS elements. Exceptions were the S. enterica Tennessee isolates whose genomes do not appear to contain an IS element upstream of the lac region (lac1 region of isolates 50260 and 50261) and the E. coli isolates that do not harbor IS elements at either end of the lac region. All of the S. enterica subsp. enterica isolates included in this study possess an IS1 element downstream of the lacA remnant, however, different IS elements are found at the $3^{\prime}$ junction of some of the other species included in this study. The type of IS element located at the $5^{\prime}$ junction of the lac region is more variable. It appears that the lac region is carried on several different transposable elements among Enterobacteriaceae. It was determined that the lac region found in the genomes of 
Table 1 Genetic organization and genomic location of lac and flanking region for Salmonella isolates and other Enterobacteriaceae

\begin{tabular}{|c|c|c|c|c|c|c|c|}
\hline Accession no. & Isolate & Location & $5^{\prime}$ & $\operatorname{alg} L^{b}$ & laclZY & $\operatorname{lac}^{\mathrm{c}}$ & $3^{\prime}$ \\
\hline JZTM00000000 & S. enterica Montevideo 50262 & plasmid & IS903D & + & + & $\mathrm{t}$ & IS1 \\
\hline JZTT00000000 & S. enterica Montevideo 50270 & plasmid & IS903D & + & + & $\mathrm{t}$ & IS1 \\
\hline JZTN00000000 & S. enterica Senftenberg 50263 & plasmid & IS903D & + & + & t & IS1 \\
\hline JZTP00000000 & S. enterica Senftenberg 50265 & plasmid & IS903D & + & + & $\mathrm{t}$ & IS1 \\
\hline JZTU00000000 & S. enterica Senftenberg 50271 & plasmid & IS903D & + & + & t & IS1 \\
\hline JZTV00000000 & S. enterica Senftenberg 50272 & plasmid & IS903D & + & + & $\mathrm{t}$ & IS1 \\
\hline JZTO00000000 & S. enterica Senftenberg 50264 & plasmid & IS903D & + & + & $\mathrm{t}$ & IS1 \\
\hline EF382672 & K. pneumoniae pK29 & plasmid & IS903D & + & + & $\mathrm{t}$ & IS1 \\
\hline JZTS00000000 & S. enterica Indiana 50269 & chromosome & transposon & ++ & + & $\mathrm{t}$ & IS1 \\
\hline СР006731 & C. sakazakii CMCC 45402 & chromosome & IS5075 & ++ & + & t & IS1 \\
\hline СР010377 & E. cloacae 34983 & chromosome & IS5075 & ++ & + & $\mathrm{t}$ & IS1 \\
\hline CP004091 & C. sakazakii SP291 & chromosome & ISEhe $^{\mathrm{a}}$ & - & + & $\mathrm{t}$ & $\mid S 1^{a}$ \\
\hline CP001918 & E. cloacae ATCC 13047 & chromosome & ISEhe $^{\mathrm{a}}$ & - & + & $\mathrm{t}$ & $\mid S 1^{a}$ \\
\hline CP008842 & K. oxytoca pKOXM1A & plasmid & $1 S 26$ & $t^{a}$ & + & - & IS903D \\
\hline СР006927 & K. pneumoniae p30660_1 & plasmid & IS5075 & ++ & + & - & IS903D \\
\hline СР000648 & K. pneumoniae pKPN3 & plasmid & IS5075 & ++ & + & - & IS903D \\
\hline JX442974 & K. pneumoniae pKN-LS6 & plasmid & ISEC $8^{\mathrm{a}}$ & ++ & + & - & IS903D \\
\hline JN233704 & K. pneumoniae pKPN-IT & plasmid & ISEc $8^{\mathrm{a}}$ & ++ & + & - & IS903D \\
\hline CP006657 & K. pneumoniae $\mathrm{p} 1$ & plasmid & ISEC $8^{\mathrm{a}}$ & ++ & + & - & IS903D \\
\hline F0834905 & K. pneumoniae pKP52.145_॥ & plasmid & 152 & ++ & + & t & $\mid S 1^{a}$ \\
\hline$J \times 424424$ & K. pneumoniae pKPN_CZ & plasmid & IS5075 & ++ & + & t & IS1 \\
\hline KF719971 & K. pneumoniae pKP007 & plasmid & ISEC $8^{\mathrm{a}}$ & ++ & + & $\mathrm{t}$ & $|S|$ \\
\hline CP008843 & K. oxytoca pKOXM1B & plasmid & iso-IS1 & - & + & - & IS5 \\
\hline HF571988 & Y. enterocolitica YE53/03 & chromosome & 152 & - & + & $\mathrm{t}$ & IS1 \\
\hline JZTK00000000 & S. enterica Tennessee 50260 lac2 & chromosome & ISEC $8^{\mathrm{a}}$ & ++ & + & $\mathrm{t}$ & IS1 \\
\hline JZTL00000000 & S. enterica Tennessee 50261 lac2 & chromosome & ISEC $8^{\mathrm{a}}$ & ++ & + & $\mathrm{t}$ & IS1 \\
\hline JZTJ00000000 & S. enterica Tennessee 50259 & chromosome & umuD & - & + & t & IS1 \\
\hline JZTK00000000 & S. enterica Tennessee 50260 lac1 & chromosome & umuD & - & + & $\mathrm{t}$ & IS1 \\
\hline JZTL00000000 & S. enterica Tennessee 50261 lac1 & chromosome & umuD & - & + & t & IS1 \\
\hline AHUY01000000 & S. enterica Tennessee 4535 & chromosome & umuD & - & + & t & IS1 \\
\hline СР009855 & E. cloacae pENT-22e & plasmid & ISEC $8^{\mathrm{a}}$ & ++ & + & $\mathrm{t}$ & IS1 \\
\hline СР009866 & Pantoea sp. PSNIH2 & chromosome & acetyltransferase & - & + & $\mathrm{t}$ & IS1 \\
\hline BA000007 & E. coli Sakai & chromosome & $m h p R$ & - & + & + & $\operatorname{cyn} X$ \\
\hline AE014075 & E. coli CFT073 & chromosome & yail & - & + & + & $\operatorname{cod} A$ \\
\hline FN554766 & E. coli 042 & chromosome & $m h p R$ & - & + & + & $\operatorname{cyn} X$ \\
\hline CP003289 & E. coli 2011 C-3493 & chromosome & $m h p R$ & - & + & + & $\operatorname{cyn} x$ \\
\hline U00096 & E. coli K-12 MG1655 & chromosome & $m h p R$ & - & + & + & $\operatorname{cyn} X$ \\
\hline СР000880 & S. enterica arizonae RSK2980 & chromosome & $a c n B$ & - & + & + & speD \\
\hline JZTQ00000000 & S. enterica diarizonae 50267 & chromosome & $a c n B$ & - & + & + & speD \\
\hline JZTR00000000 & S. enterica diarizonae 50268 & chromosome & $a c n B$ & - & + & + & speD \\
\hline
\end{tabular}

aremnant

balginate lyase gene upstream of lacl: 897 bp, +; 936 bp, ++; negative, -

truncated, $t$; negative, $-;$ positive, + 
the S. enterica subsp. diarizonae isolates is not flanked by IS elements on either side.

Interestingly, characterization of the genome upstream of the lac region revealed an additional gene transcribed in the opposite direction of the lac genes between the IS element and lacI for the S. enterica isolates included in this study except isolate 50259, the lac1 region in isolates 50260 and 50261, and the S. enterica subsp. diarizonae isolates. This gene was found in the same genetic location in some of the other enteric bacterial species included in this study. BLASTp analysis revealed that the protein encoded by this gene has $99 \%$ sequence identity to alginate lyase [GenBank:WP_012477405] encoded in the Klebsiella pneumoniae genome. For three of the $S$. enterica isolates, 50260, 50261, and 50269, this gene is $39 \mathrm{bp}$ longer and the protein includes an additional oxidoreductase region, resulting in the highest protein sequence identity to a bifunctional oxidoreductase/alginate lyase found in Enterobacteriaceae [GenBank:WP_032414406]. The presence or absence of this additional gene along with the gene length if present is noted in Table 1.

\section{Genomic location of lac operon}

We sought to determine where the mobile elements carrying the lac region were inserted in the S. enterica genomes. The lac regions of several of the S. enterica isolates in this study displayed a high sequence similarity and flanking organization with that of the lac region on plasmid pK29 harbored in K. pneumoniae strain NK29 [GenBank:EF382672]. Plasmid pK29 is 269,674 bp in size and the flanking organization is included in Table 1. The sequencing reads from the $11 \mathrm{~S}$. enterica subsp. enterica isolates in this study were mapped to the plasmid pK29 reference sequence. The mappings demonstrated that seven of the isolates, namely Montevideo isolates 50262 and 50270 and Senftenberg isolates 50263, 50264, 50265, 50271 and 50272, harbor a plasmid with similarity to $\mathrm{pK} 29$. The consensus sequences resulting from the mappings revealed $99 \%$ identity to pK29. Furthermore, BLAST comparisons revealed that these seven strains contain the repHI2 and repA genes that encode replication proteins found in the pK29 plasmid sequence. Gaps in the $S$. enterica plasmid sequences were primarily due to the presence of regions containing a total of six antibiotic resistance genes in pK29. However, isolate 50271 was missing only three of the resistance genes. Although it was determined that the consensus from the mapping was $\sim 20.8 \mathrm{~kb}$ shorter than pK29, it is not known whether there are additional insertions in the plasmids harbored by these seven S. enterica isolates.

The lac region of Tennessee isolate 50259 and the lac1 regions of Tennessee isolates 50260 and 50261 exhibited identical flanking organization and sequence with that of the lac region harbored on the chromosome of the S. enterica Tennessee isolate responsible for an outbreak associated with contaminated peanut butter [GenBank:AHUY01000000]. Genome comparisons confirmed that the lac regions harbored in the Tennessee isolates included in this study (lac1 regions for 50260 and 50261) are carried in the chromosome at the same location, within a $72 \mathrm{~kb}$ island inserted at $p h e V$, as the Tennessee peanut butter outbreak isolate. Despite the similarity in flanking organization between the lac2 regions harbored in the genomes of isolates 50260 and 50261 with those found on two different plasmids, pKP007 and pENT-22e, carried in other enteric species (Table 1), the lacIZY sequences are not similar to those on the plasmids and sequencing read mappings determined that both isolates lack these plasmids. The lac2 regions appear to be located on the chromosome in an unknown location as both plasmid extractions and BLAST searches of the genome sequences for an origin of replication failed to reveal the presence of a plasmid in these isolates. The lac region was determined to be chromosomal for the Indiana isolate 50269. It is located within a $95 \mathrm{~kb}$ island inserted in the $y g i R$ gene. Also chromosomally located, the lac region found in the $S$. enterica subsp. diarizonae isolates is inserted between the $a c n B$ and speD genes.

\section{Phylogenetic analysis of the laclZY region}

Along with characterizing the lac regions and flanking sequence in the genomes, we determined the sequence similarity of the concatenated lacI, lacZ, and lacY genes of the S. enterica isolates and other enteric bacterial species (Fig. 1). As expected, the lac genes carried by the Senftenberg and Montevideo isolates (50262-50265, 50270-50272) displayed a high degree of similarity (99.98\%) with those found on the K. pneumoniae plasmid pK29 (Additional file 1 Figure S1). In general, similar lac region flanking organization corresponds to highest lac gene sequence identity. One notable exception is the lac 2 region in the Tennessee isolates 50260 and 50261 where the flanking IS elements, as well as the lengths of the gene upstream of lacI and the lacA remnant, are similar to the K. pneumoniae plasmid pKP007, but the lacIZY gene sequences share only $98 \%$ identity. The S. enterica subsp. diarizonae lac gene sequences share low (73 \%) homology with those of the S. enterica subsp. enterica serovars and other Enterobacteriaceae. Clearly, the lac gene sequences do not cluster according to plasmid or chromosomal location (Table 1, Fig. 1).

\section{MLST analysis of Salmonella isolates}

MLST analysis of the Salmonella isolates was performed using the aroC, dnaN, hemD, hisD, purE, sucA, and thrA loci (Fig. 2). Isolates belonging to the same serovar had identical sequence types (STs) regardless of the presence 


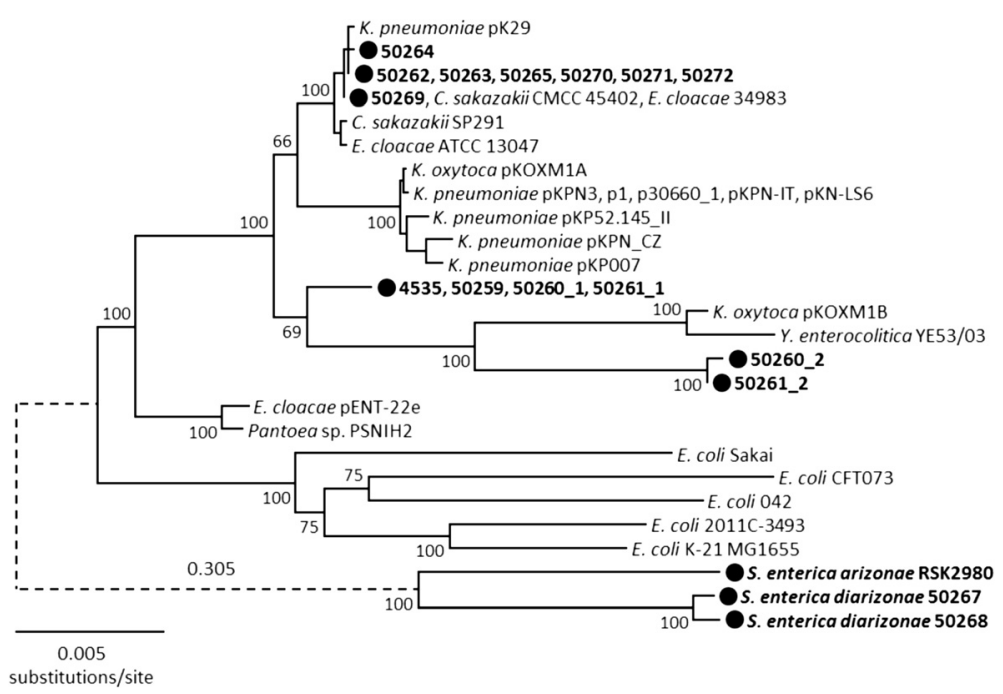

Fig. 1 Phylogenetic relationships of laclZY for Salmonella isolates and other Enterobacteriaceae. This neighbor-joining tree was constructed using the Kimura two-parameter model of nucleotide substitution. Bootstrap values based on 500 replications are given at the internal nodes. Salmonella isolates are indicated by the black circles and bold text. The branch connecting the $S$. enterica subsp. arizonae/diarizonae cluster with the rest of the isolates was shortened to improve the clarity of the relationships and its actual length is given above the dashed line

or absence of the lac genes, suggesting multiple horizontal transfer events occurred to give rise to the Lac + isolates. Observed STs among the S. enterica subsp. enterica isolates include ST14 (Senftenberg), ST17 (Indiana), ST316 (Montevideo), and ST319 (Tennessee). The Lac- closed genome strains Enteritidis P125109 (ST11), Typhimurium LT2 (ST19), and Typhi CT18 (ST2) were included in the analysis for reference. Among the $S$. enterica subsp. diarizonae strains, isolate 50267 was found to possess ST1261, while isolate 50268 has a variant of ST432 due to a single $\mathrm{T}$ to $\mathrm{C}$ synonymous transition in sucA. The S. enterica subsp. arizonae closed genome strain RSK2980 was also included for reference as it rooted the MLST phylogeny. RSK2980 has a novel ST comprised of previously observed alleles. Its allele profile of aroC65, dnaN25, hemD29, hisD24, purE20, sucA50, and thrA497 is most similar to ST1430, differing by 7 polymorphic sites ( 1 in $\operatorname{dnaN,} 5$ in hisD, and 1 in thrA).

\section{Discussion}

Strains of S. enterica are commonly regarded as nonlactose fermenting pathogens; however a small percentage of isolates have acquired the lac region which can lead to confusion from a diagnostic standpoint. The lac operon has been well studied in E. coli and is known to be chromosomally located. However it has been noted some time ago that the lac operon is carried on a plasmid in some Enterobacteriaceae species including $S$. enterica Typhimurium and Oranienburg isolates [12]. Subsequent to that report, it was determined that the lac

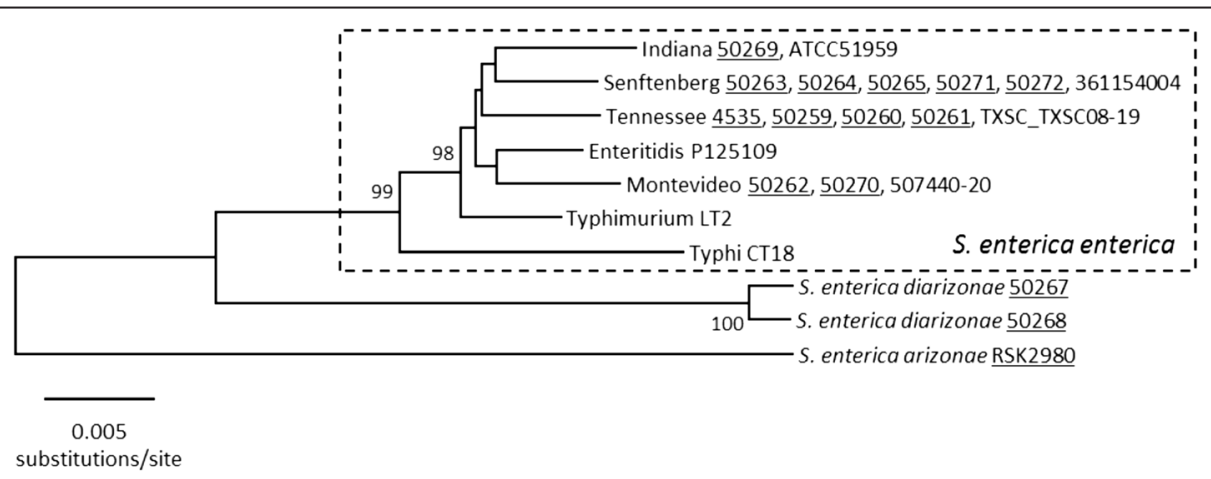

Fig. 2 MLST analysis of Salmonella isolates. This neighbor-joining tree was constructed using the Kimura two-parameter model of nucleotide substitution. Bootstrap values $>90 \%$ based on 500 replications are given at the internal nodes. S. enterica subsp. enterica isolates are enclosed by the dashed box. Lac + isolates are underlined. The Lac- closed genome strains (Enteritidis P125109, Typhimurium LT2, and Typhi CT18) are included for reference 
operon was located on plasmids harbored in S. enterica Typhi isolated from an individual with typhoid fever [14] and S. enterica Newport responsible for an outbreak in a nursing home [5]. In contrast, it was shown that the lac operon was not carried on a plasmid in an S. enterica Indiana isolate [13]. We found that the lac region harbored in the Indiana isolate included in this study is also chromosomally located, as are the lac regions carried in the $S$. enterica Tennessee genomes. The remaining S. enterica subsp. enterica isolates in this study possess the lac genes on a plasmid similar to K. pneumoniae pK29. Interestingly, despite their chromosomal location, the lac genes harbored in the Indiana isolate also display high sequence similarity with the lac genes found on pK29. Our results revealed other instances of lac genes with high sequence similarity but different genomic location (Table 1, Fig. 1); namely, Enterobacter cloacae [GenBank:CP009855] and Pantoea sp. [GenBank:CP009866] as well as Klebsiella oxytoca [GenBank:CP008843] and Y. enterocolitica [GenBank:HF571988]. Clearly, the Lac + S. enterica isolates harboring a chromosomal lac region are not isolates in which the lac operon was maintained in the S. enterica genome as is thought to be the case for $E$. coli. The most likely explanation for this observation is that $S$. enterica and $E$. coli diverged from a common ancestor and that the lac operon was either deleted from S. enterica or gained by $E$. coli as suggested by Riley [19].

In fact, we found that two of the S. enterica Tennessee isolates in this study possess two lac regions each having different sequence homology, flanking organization, and genomic location, none of which is similar to the lac region in E. coli. Furthermore, our results are consistent with a lac region that is highly mobile among Enterobacteriaceae as it is almost always flanked by IS elements and this entire region is often inserted in another mobile element, namely a plasmid or chromosomal island. Also, there is a greater correlation between lac gene sequence and flanking organization than with genomic location or bacterial species. The combined analysis of genetic organization, MLST, lac gene sequence homology, and genomic location in this study clearly demonstrate that S. enterica subsp. enterica has acquired the lac region multiple times through independent events.

The ability to ferment lactose can offer bacteria a growth advantage in environments where lactose is present such as the intestine. Wilkins and Franzese discovered that in a competition experiment utilizing gnotobiotic mice, a lactose-fermenting E. coli strain grew to a level ten times greater than the isogenic lactose negative strain [20]. A screen of $552 \mathrm{~S}$. enterica isolates from dried milk products or milk-drying plants revealed $15.6 \%$ were $\mathrm{Lac}+[10]$, a higher percent than the $0.8 \%$ $\mathrm{Lac}+\mathrm{S}$. enterica reported in a Centers for Disease Control and Prevention (CDC) survey of 371 cultures [4].
This suggests the readily available lactose in the environment played a selective role for lactose fermenting ability. Interestingly, S. enterica Tennessee strain 50261 was isolated from an environment containing dry milk. The other Tennessee isolates in this work were isolated from peanut butter; however source information is not available for many of the isolates in this study so it is unclear how many others may have been associated with a lactose-rich environment.

While lactose fermentation could provide a fitness advantage to $S$. enterica in the environment outside the vertebrate host and in the host gut, it has been reported that the lacI and lacA genes may act as antivirulence factors by attenuating proliferation inside macrophages or invasion of epithelial cells, respectively, in the host [21-23]. Eswarappa and colleagues demonstrated that, inside macrophages, expression of lacI from a plasmid in an S. enterica Typhimurium strain missing the lac operon causes down regulation of three genes on the SPI-2 pathogenicity island [21]. However, others found no influence on invasion of epithelial cells by a Typhimurium strain harboring a LacI-expressing plasmid [23]. Rather, they concluded that the lacA gene, to the same extent as the entire lac operon, significantly inhibited transcription of four flagellar genes, thus decreasing epithelial cell invasion [23]. This is consistent with previous experiments utilizing a Typhimurium strain in which it was determined that flagella are required for full invasive potential in a tissue culture invasion assay as well as for a complete inflammatory response in the calf intestine [24].

Although the acquisition of the lac operon by a Salmonella isolate can be beneficial in some environments, in others it would be a metabolic burden, thus regulation via the LacI protein would be important. Contrary to the assertion that Lac + S. enterica strains lack lacI [21], we have found that Lac $+S$. enterica carry an intact lacI repressor gene. The presence of an intact lacI gene in S. enterica has also been reported by others [12, 14]. Instead, our results reveal a truncated lacA gene in Lac + S. enterica. Certainly, $\mathrm{Lac}+$ S. enterica with intact lacI, but truncated lacA, have caused gastroenteritis in humans. The truncated $l a c A$ we noted carried by all the $S$. enterica subsp. enterica isolates in this study resulted in a considerably smaller 56 amino acid protein rather than the full length 220 amino acid $E$. coli LacA, therefore it is highly likely it does not exert the effect of repressing the flagellar genes. Macrophage assays may be a superior model for systemic S. enterica infections, while epithelial cell invasion assays have been shown to be better models for enteritis in humans since some virulence determinants required for growth at systemic sites such as SPI-2 are less important during an infection localized in the intestinal epithelia [24, 25]. Our genomic characterization results are consistent with the fact that since almost all S. enterica subsp. enterica are localized to 
the intestinal tract in the host, lacI may not play a role as an antivirulence factor in these strains, while lacA appears to be an important antivirulence factor candidate.

\section{Conclusions}

Altogether, our results suggest that the lac region is highly mobile among Enterobacteriaceae and the Lac + S. enterica subsp. enterica serovars have acquired the lac region through parallel events. The argument could be made that the loss of the lac region was originally driven by the adaptation of $S$. enterica as it evolved to become an invasive pathogen. However, some isolates have now reacquired a version of the lac region that possesses a truncated lacA gene as this would allow the pathogen to utilize lactose when advantageous but not inhibit invasion.

\section{Methods}

Bacterial strains, culture conditions, and DNA isolation The 13 Salmonella enterica isolates 50259-50265 and 50267-50272 sequenced in this study were kindly provided by Rebecca Dievart. The sequenced isolates along with all other bacteria that were in included in the genetic analysis performed in this study are listed in Table 1. Overnight cultures of the sequenced isolates were grown in Luria broth at $37^{\circ} \mathrm{C}$. The lactose-fermenting phenotype was determined using cultures grown on MacConkey agar plates incubated at $37^{\circ} \mathrm{C}$ for $24 \mathrm{~h}$. Genomic DNA was extracted from overnight cultures using the DNeasy Blood and Tissue Kit (Qiagen, Germantown, MD, USA).

\section{Whole genome sequencing}

Sequencing libraries were prepared from genomic DNA with the TruSeq DNA Sample Prep Kit (Illumina, San Diego, CA, USA) and sequenced on the Illumina MiSeq Platform, generating paired-end $250 \mathrm{bp}$ reads in sufficient quantity to provide between $42 \mathrm{X}$ and $303 \mathrm{X}$ coverage for each genome. Raw reads were trimmed and draft genome sequences were assembled de novo with CLC Genomics Workbench v6.5.1 or v7.0.3 (CLC bio, Boston, MA, USA).

\section{Molecular serotyping}

The molecular serotypes of the S. enterica isolates were determined from the draft genomes by BLAST analysis using the $w z x, w z y, w z m, w z t, w b b E$, wbbF, wbaV, weiD, $o a c, f l i C, f l j B$, and $f l p A$ loci.

Characterization of the lac region and location in genome The lac genes were found in the draft genomes by BLAST using the sequence of the E. coli strain K-12 MG1655 operon [GenBank:U00096, locus tags b0342b0345] as a query. In most cases the entire lac operon was contained on one contig; otherwise two contigs were bioinformatically joined to obtain the entire lac operon sequence. The joined sequence was then verified by mapping the reads onto the joined sequence using CLC Genomics Workbench (CLC bio). The sequence regions flanking the operons were characterized by BLAST analysis. To determine the location of the lac operons within the genome, the lac region was used as a BLAST query, followed by identification of the flanking genes by BLAST. The locations of lac operon regions in bacterial species with the highest sequence identity were examined for similarity with the S. enterica genomes in this study.

\section{Phylogenetic analyses}

DNA sequences of the lacIZY region $(\sim 5.7 \mathrm{~kb})$ of the operons were combined with those of selected $l a c+$ bacterial species from GenBank and aligned with the ClustalW algorithm using the MegAlign module of the Lasergene software package (DNAStar Inc., Madison, WI). A pairwise similarity matrix of the lacIZY sequences analyzed is provided as supplemental material. In silico multilocus sequence typing (MLST) was performed on seven conserved housekeeping genes (aroC, dnaN, hemD, hisD, purE, sucA, and $t h r A$ ) [26] through extraction of the relevant sequences from the draft genome assemblies of the strains investigated in this study. These sequences, along with those of selected Salmonella isolates from GenBank (AE006468, AL513382, AM933172, CP000880, CP007505, CP007530, AHUY01000000, AOZC01000000, and AYDO 01000000) were concatenated for phylogenetic analysis. Allele and sequence type designations were determined via the Salmonella MLST database website (http:// mlst.warwick.ac.uk/mlst/dbs/Senterica). Neighbor-joining trees were constructed using the Kimura two-parameter model of nucleotide substitution with the MEGA3 software [27], and the inferred phylogenies were tested with 500 bootstrap replications for both the lacIZY and MLST sequences.

\section{Nucleotide sequence accession numbers}

The draft genome sequences of the $S$. enterica strains 50259, 50260, 50261, 50262, 50263, 50264, 50265, 50267, 50268, 50269, 50270, 50271, and 50272 were deposited at DDBJ/EMBL/GenBank under accession nos. JZTJ000000 00, JZTK00000000, JZTL00000000, JZTM00000000, JZTN 00000000, JZTO00000000, JZTP00000000, JZTQ0000000 0, JZTR00000000, JZTS00000000, JZTT00000000, JZTU0 0000000 and JZTV00000000, respectively.

\section{Additional file}

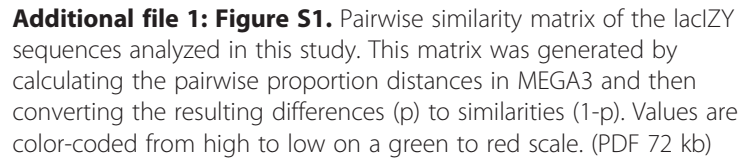

Additional file 1: Figure S1. Pairwise similarity matrix of the laclZY sequences analyzed in this study. This matrix was generated by calculating the pairwise proportion distances in MEGA3 and then converting the resulting differences $(p)$ to similarities (1-p). Values are color-coded from high to low on a green to red scale. (PDF 72 kb) 


\section{Competing interests}

The authors declare that they have no competing interests.

\section{Authors' contributions}

SRL performed genome sequencing, contributed to analysis and interpretation of the results, and drafted the manuscript. DWL analyzed and interpreted results and revised the manuscript. KAL conceived the study and revised the manuscript. All authors read and approved the final manuscript.

\section{Acknowledgements}

We thank Rebecca Dievart at BIO-RAD Laboratories, France, for the Lac + Salmonella isolates. One of us (KAL) is indebted to Monica Riley whose work on genome comparisons decades ago inspired this work. This research was funded in part by the Oak Ridge Institute for Science Education.

\section{Received: 14 April 2015 Accepted: 17 August 2015}

Published online: 25 August 2015

\section{References}

1. Gal-Mor O, Boyle EC, Grassl GA. Same species, different diseases: how and why typhoidal and non-typhoidal Salmonella enterica serovars differ. Front Microbiol. 2014;5:391.

2. Coburn B, Grassl GA, Finlay BB. Salmonella, the host and disease: a brief review. Immunol Cell Biol. 2007;85(2):112-8.

3. Fookes M, Schroeder GN, Langridge GC, Blondel CJ, Mammina C, Connor $\mathrm{TR}$, et al. Salmonella bongori provides insights into the evolution of the Salmonellae. PLoS Pathog. 2011;7(8):e1002191.

4. Ewing WH. Differentiation of Enterobacteriaceae by biochemical reactions. Atlanta: Center for Disease Control; 1973.

5. Anand CM, Finlayson MC, Garson JZ, Larson ML. An institutional outbreak of Salmonellosis due to lactose-fermenting Salmonella newport. Am J Clin Pathol. 1980;74(5):657-60.

6. Dube SD. Outbreak of food poisoning caused by lactose-fermenting Salmonella tuebingen. J Clin Microbiol. 1983;17(4):698-9.

7. Farley JD, Ghesquiere W, MacLean DR, Moulton GS. Endemic institutional salmonellosis due to lactose-fermenting Salmonella newport in Nova Scotia. CMAJ. 1988;138(5):434-6.

8. Deng X, Salazar JK, Frezet S, Maccannell D, Ribot EM, Fields PI et al. Genome Sequence of Salmonella enterica Serotype Tennessee Strain CDC07-0191, Implicated in the 2006-2007 Multistate Food-Borne Outbreak Linked to Peanut Butter in the United States. Genome Announc. 2013, 1(3)

9. Gonzalez AB. Lactose-fermenting Salmonella. J Bacteriol. 1966;91(4):1661-2.

10. Blackburn BO, Ellis EM. Lactose-fermenting Salmonella from dried milk and milk-drying plants. Appl Microbiol. 1973;26(5):672-4.

11. Falcao DP, Trabulsi LR, Hickman FW, Farmer 3rd JJ. Unusual Enterobacteriaceae: lactose-positive Salmonella typhimurium which is endemic in Sao Paulo, Brazil. J Clin Microbiol. 1975;2(4):349-53.

12. Guiso N, Ullmann A. Expression and regulation of lactose genes carried by plasmids. J Bacteriol. 1976;127(2):691-7.

13. Hall ML, Threlfall EJ, Rowe B, Pinegar JA, Gibson GL. Lactose-fermenting Salmonella indiana from turkeys in Britain. Lancet. 1978;2(8101):1197-8.

14. Ezaki T, Liu SL, Yabuuchi E, Sasakawa C, Yoshikawa M. Molecular characterization of a conjugative R-lac plasmid in Salmonella typhi isolated from a patient with typhoid fever. Ann Inst Pasteur Microbiol. 1987;138(3):303-11.

15. McDonough PL, Shin SJ, Lein DH. Diagnostic and public health dilemma of lactose-fermenting Salmonella enterica serotype Typhimurium in cattle in the Northeastern United States. J Clin Microbiol. 2000;38(3):1221-6.

16. Kumar R, Surendran PK, Thampuran N. Detection and characterization of virulence factors in lactose positive and lactose negative Salmonella serovars isolated from seafood. Food Control. 2009;20:376-80.

17. Cornelis G, Ghosal D, Saedler H. Tn951: a new transposon carrying a lactose operon. Mol Gen Gen. 1978;160(2):215-24.

18. Popoff MY, LeMinor LE. Salmonella. In: Garrity GM, editor. Bergey's Manual of Systematic Bacteriology. 2nd ed. New York: Springer Science; 2005.

19. Lampel KA, Riley M. Discontinuity of homology of Escherichia coli and Salmonella typhimurium DNA in the lac region. Mol Gen Gen. 1982;186(1):82-6.

20. Wilkins TD, Franzese JA. Growth advantage of fast lactose-fermenting mutants of slow lactose-fermenting coliform bacteria in gnotobiotic rats and mice. Infect Immun. 1971;4(6):780-2.
21. Eswarappa SM, Karnam G, Nagarajan AG, Chakraborty S, Chakravortty D. lac repressor is an antivirulence factor of Salmonella enterica: its role in the evolution of virulence in Salmonella. PLoS One. 2009;4(6).

22. Bliven KA, Maurelli AT. Antivirulence genes: insights into pathogen evolution through gene loss. Infect Immun. 2012;80(12):4061-70.

23. Jiang $L$, Ni Z, Wang $L$, Feng $L$, Liu B. Loss of the lac operon contributes to Salmonella invasion of epithelial cells through derepression of flagellar synthesis. Curr Microbiol. 2015;70(3):315-23.

24. Schmitt CK, Ikeda JS, Darnell SC, Watson PR, Bispham J, Wallis TS, et al. Absence of all components of the flagellar export and synthesis machinery differentially alters virulence of Salmonella enterica serovar Typhimurium in models of typhoid fever, survival in macrophages, tissue culture invasiveness, and calf enterocolitis. Infect Immun. 2001;69(9):5619-25.

25. Santos RL, Zhang S, Tsolis RM, Kingsley RA, Adams LG, Baumler AJ. Animal models of Salmonella infections: enteritis versus typhoid fever. Microbes Infect/Institut Pasteur. 2001;3(14-15):1335-44.

26. Achtman M, Wain J, Weill FX, Nair S, Zhou Z, Sangal V, et al. Multilocus sequence typing as a replacement for serotyping in Salmonella enterica. PLoS Pathog. 2012;8(6):e1002776.

27. Kumar S, Tamura K, Nei M. MEGA3: Integrated software for Molecular Evolutionary Genetics Analysis and sequence alignment. Brief Bioinform. 2004;5(2):150-63.

\section{Submit your next manuscript to BioMed Central and take full advantage of:}

- Convenient online submission

- Thorough peer review

- No space constraints or color figure charges

- Immediate publication on acceptance

- Inclusion in PubMed, CAS, Scopus and Google Scholar

- Research which is freely available for redistribution 